Article

\title{
Joint AP Association and Bandwidth Allocation Optimization Algorithm in High-Dense WLANs
}

\author{
Jianjun Lei *, Jiarui Tao and Shanshan Yang \\ School of Computer Science and Technology, Chongqing University of Posts and Telecommunications, \\ Chongqing 400065, China; taojiarui@outlook.com (J.T.); shanshan.yang@itel-mobile.com (S.Y.) \\ * Correspondence: leijj@cqupt.edu.cn; Tel.: +86-139-9635-3443
}

Received: 12 June 2018; Accepted: 3 August 2018; Published: 6 August 2018

\begin{abstract}
Regarding access point (AP) overload and performance anomaly which is caused by mobile terminals with different bitrates, a joint AP association and bandwidth allocation optimization algorithm is presented in this paper. Meanwhile, load balancing and proportional fairness are analyzed and formulated as an optimization model. Then, we present a Fair Bandwidth Allocation algorithm based on clients' Business Priority (FBA-BP), which allocates bandwidth based on the bandwidth demand of clients and their business priority. Furthermore, we propose a Categorized AP Association algorithm based on clients' demands (CAA-BD), which classifies APs by different types of clients and chooses an optimal associating AP for a new client according to AP categories and the aggregated demand transmission time that are calculated by the FBA-BP algorithm. The CAA-BD can achieve load balance and solve the performance anomaly caused by multi-rate clients coexisting. The simulation results show that our proposed algorithm obtains significant performance in terms of AP utilization, throughput, transmission delay and channel fairness in different client density levels compared with the categorized and Strong Signal First (SSF) algorithms.
\end{abstract}

Keywords: AP association; bandwidth allocation; load balancing; WLANs

\section{Introduction}

In recent years, the characteristics of IEEE 802.11 Wireless Local Area Networks (WLANs), such as its easy deployment, flexibility, and low cost, have made it a popular connectivity method to access the Internet. The IEEE 802.11 standards use the Received Signal Strength Indicator (RSSI) as the access point (AP) association scheme, which may lead the stations (STAs) to make associations with congested APs, while leaving adjacent APs to carry very light load or even to be idle. This kind of load unbalancing could result in network performance declining. Therefore, AP association strategy is crucial for load balance. However, various adopted metrics of AP association such as the traffic of clients [1,2], the number of associated clients [3], and the channel busy ratio [4] still cannot refrain AP performance degradation. In addition, the MAC protocol of 802.11 based on DCF (Distributed Coordination Function) provides equal long-term transmission opportunities to all clients associated with the same AP [5]. In multi-rate WLANs, due to this kind of throughput-based fairness scheme, clients with lower rates occupy the channel for a longer time than those with higher rates do, which leads to the unfairness of channel access among clients and suppresses the aggregated throughput. Besides, the types of application services are diversified in various wireless terminals, and the different types of clients have different requirements for Quality of Service (QoS) [6], such as bandwidth and delay. Nevertheless, almost all proposed AP bandwidth allocation strategies are based on the assumption that any client has an enormous bandwidth demand; therefore, they cannot be satisfied no matter how the bandwidth of APs is allocated to them. The flexible AP bandwidth 
allocation strategy based on their demand and business priority can provide more diverse service and thus perhaps bring the user a better experience.

In this paper, AP association and bandwidth allocation are jointly considered as the constraints of channel access for achieving load balance and proportional fairness in multi-rate WLANs scenario. We firstly present a Fair Bandwidth Allocation strategy based on clients' Business Priority (FBA-BP), in which the clients' bandwidth is calculated based on their demand and business priority. In addition, then the aggregated transmission time demanded by all the clients will be regarded as the metric of AP load. Consequently, we propose a Categorized AP Association algorithm Based on the Demands of clients (CAA-BD), which chooses the optimal associating AP according to its category and the aggregated transmission time demanded by all the associating clients. Eventually, these two joint algorithms can realize the proportional fairness of channel access and resolve the performance anomaly, thus bringing significant throughput improvement, especially in high-dense scenarios. The basic idea of AP associating algorithm was first published in CSOC2017 [7]. However, our new proposed CAA-BD is considering not only clients' data rates but also their business demand as the associating metric of the AP, and we also present a more detailed analytical model in the new scheme. Finally, the joint $\mathrm{AP}$ association and bandwidth allocation can conduct a more delicate AP selection and channel allocation, and the evaluation experiments also demonstrate that the more significant performance can be obtained in terms of AP utilization and channel fairness index.

The remainder of the paper is organized as follows. We give some related works in Section 2. Section 3 provides a motivation model and theoretical analysis. Section 4 presents the details of FBA-BP and CAA-BD. We give the simulation results in Section 5 and conclude this paper in Section 6.

\section{Related Work}

Since the conventional RSSI-based AP association scheme may lead to imbalanced load traffic among APs and unfairness bandwidth allocation among clients, many literatures concentrate on conceiving AP association strategies as the alternative of the default Strong Signal First (SSF) scheme in legacy IEEE802.11.

There have been several AP association schemes for load balance among APs and various metrics are first studied to determine the AP load in some literatures. In [8], energy consumption and throughput are both proposed as the indication of AP load. In [9], the author uses the file download time for web browsing to estimate the AP load. In [10], the traffic intensity of clients and the effect of hidden terminals are considered as the main factors of AP load. Simultaneously, many load balancing strategies for AP association are also investigated. In [11], Chen et al., propose a load balancing strategy for AP association based on the game theory with local information. In [12], a multi-constraint load balancing scheme based on cell breathing is utilized for the tradeoff between load balancing among APs and data power loss of clients. However, they are infeasible in many realistic environments due to their high complexity for clients. Gong et al. [13] proposes a distributed adaptive load balancing algorithm for multi-rate WLANs, which uses some innovative load metrics such as throughput and transmission rates. However, it ignores the negative influence of newly arriving clients on the throughput of the existing clients.

In [14], a comprehensive study about the correlation between load balancing and channel fairness is presented, which indicates that they cannot be easily achieved simultaneously. In [15], Li et al., propose a time-based fair AP algorithm, which jointly considers power control and AP association for aggregated throughput and proportional fairness. However, in this algorithm, the AP association is formulated as a NP-hard problem, which can lead the clients to switch among APs frequently due to its multiple iteration process often triggering the power adjustment. In [16], Gong and Yang present an AP association algorithm, which estimates the throughput of clients from downlink and uplink by a bi-dimensional Markov model and can achieve proportional fairness among clients. However, this algorithm has a high time complexity because some information from all nearby APs must be obtained timely. In [17], they propose another lower complexity on-line AP association 
algorithm, namely Categorized algorithm (Categorized), which achieves the proportional fairness by classifying APs based on types of clients associating with them. Although the performance anomaly problem is eliminated, load imbalance may emerge.

In this paper, we propose a CAA-BD algorithm, which utilizes a new metric of AP load, the allocated transmission time of the AP, determined by all clients associating with it, as well as the newly arriving one. Meanwhile, we present an FBA-BP algorithm, which can gather the aggregated transmission time demand according to the clients' business and their priority. Simultaneously, the APs are categorized by the types of their associating clients. Therewith, the clients will give preference to associating the AP with the same type and the least allocated transmission time. This algorithm not only improves load balance among APs, but also takes channel fairness into consideration, which effectively minimizes the impact of performance anomaly.

\section{Network Model and Design Requirements}

In what follows, we describe a network model and performance metrics, and discuss some network design requirements desired to be achieved according to the conception of our algorithms while the detailed implementation is presented in Section 4.

\subsection{Motivation}

Performance anomaly refers to the effect that, when different rate clients associating with an $\mathrm{AP}$, the throughput of the high rate clients will be degraded by the low rate clients [18], thus resulting in the decline of the system aggregated throughput. Here, a specific example is provided to demonstrate the throughput loss caused by performance anomaly. Consider a WLAN with 2 APs (AP1 and AP2) and 4 STAs, enumerated from 1 to 4, as depicted in Figure 1 [19]. STA1 and STA2 experience a bit rate of $11 \mathrm{Mbps}$ from AP1, while STA4 also experiences a bit rate of $11 \mathrm{Mbps}$ from AP2. STA3 is the newly arriving one, which is in the overlapping area between AP1 and AP2. STA3 can associate with either AP1 or AP2. If it associates with AP1, STA3 will have a bit rate of $11 \mathrm{Mbps}$, and when all clients associating with AP1 have the same bit rate, AP1 will obtain distribute bandwidth without suffering performance anomaly. However, if it chooses AP2, it will have a bit rate of $1 \mathrm{Mbps}$, and when all STAs associating with AP2 have different bit rates, AP2 will suffer performance anomaly. The analysis from the experimental data in Table 1 demonstrates the performance anomaly that is a main factor to degrade system throughput [19].

In Table 1, achievable throughputs are based on the analysis of [18], whereas those in parentheses are achieved by the simulation with OPNET. From the data of Table 1, it is noticed that though AP1 is more congested than AP2, if STA3 associates with AP1, the system throughput will be higher than that when it selects the counterpart AP2. Therefore, the bandwidth allocation scheme needs to be considered to eliminate the performance anomaly problem, preventing low rate clients from dominating the channel.

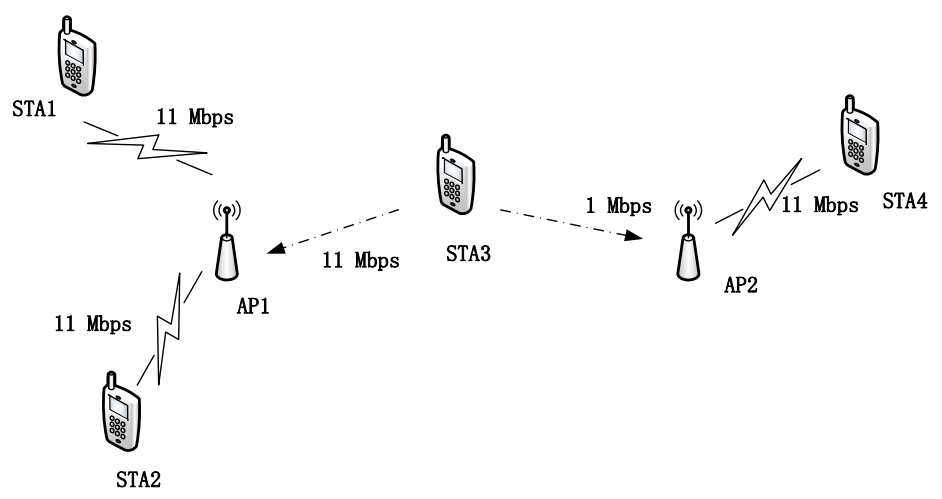

Figure 1. Scenario illustrating performance anomaly [19]. 
Table 1. Throughput Loss Caused by Performance Anomaly [19].

\begin{tabular}{cccccc}
\hline \multirow{2}{*}{ STA3's Choice } & \multicolumn{3}{c}{ Achievable Throughput (Mbps) } & \multirow{2}{*}{ System Throughput (Mbps) } \\
\cline { 2 - 5 } & STA1 & STA2 & STA3 & STA4 & \\
\hline AP1 & $4.23(3.56)$ & $4.23(3.12)$ & $4.23(3.33)$ & $11.45(8.33)$ & $24.14(18.34)$ \\
AP2 & $6.21(4.73)$ & $6.21(4.13)$ & $1.42(0.88)$ & $1.42(0.93)$ & $15.26(10.67)$ \\
\hline
\end{tabular}

\subsection{Network Model}

Our network topology models an IEEE 802.11-based multi-rate WLAN, which contains N STAs and M APs. Each AP has a limited coverage area and neighboring APs are assigned to operate at interference-free channels. Adjacent APs have overlapping coverage areas. Let A and S respectively denote the set of APs and the set of STAs. Each AP serves only STAs that reside in its coverage area. At any given time, a STA can be allowed to choose one and only one AP to associate with whereas each AP can serve multiple STAs simultaneously. The coefficient $x_{i j}$ is intended to indicate the association relationship between STA $i$ and AP $j$, which is defined as follows:

$$
x_{i j}=\left\{\begin{array}{l}
1, \text { if } \mathrm{STA} i \text { is associated } t p \mathrm{AP} j, \\
0, \text { otherwise. }
\end{array}\right.
$$

where $i=1,2, \ldots, \mathrm{N}, j=1,2, \ldots, \mathrm{M}$. It is assumed that the optimal data rate of downlink transmission can be estimated by STAs according to the RSSI of Beacon packets from every AP candidate, and it is further assumed that the channel condition between a STA and an AP is symmetric, so the optimal data rate of downlink and uplink transmissions is identical.

\subsubsection{Demands of Service}

All clients are divided into four classes according to their application businesses: voice (class-1), video (class-2), best-effort (class-3) and background (class-4) stream. The priorities of business are from the high to the low respectively. Since different application businesses have different demands of bandwidth and delay, they can be used to determine the bandwidth allocation.

\subsubsection{Transmission Time}

Transmission time demanded by client $i$ is the length of time to transmit the data that the client requires from $\mathrm{AP} j$. It is formulated as follows.

$$
T_{i j}=\frac{S_{i}}{r_{i}}=\frac{B_{i}}{r_{i}} T_{j}
$$

where, $T_{j}$ is the total transmission time of $\mathrm{AP} j$, which satisfies $T_{j}=\sum_{i=1}^{n} T_{i j}, T_{i j}$ is the transmission time demanded by client $i$ after the association is made to $\mathrm{AP} j$; $s_{i}$ is the size of data that client $i$ requires; $r_{i}$ is the transmission rate of client $i$ after the association; $B_{i}$ is the size of data required by client $i$ during per unit of time.

\subsubsection{AP Utility}

AP utility is the weighted product of the allocated data transmission time for client $i$ from AP $j$, which is related to the number of clients and the effective bandwidths and provides an efficient tradeoff between the client bandwidth and the AP load. It can be expressed by the following equation:

$$
U_{A_{j}}=\prod_{i \in A_{j}}\left(t_{i}\right)^{w_{i}}
$$


where, $A_{j}$ denotes the set of clients associated with $\mathrm{AP} j ; t_{i}$ denotes the transmission time of client $i$ obtained actually from $\mathrm{AP} j$, which satisfies the constraints of $\sum_{i=1}^{n} t_{i}=T_{j}$ and $b_{i}=\frac{r_{i} t_{i}}{T_{j}}$, where $b_{i}$ is the bandwidth that client $i$ obtains in fact from $\mathrm{AP} j$, and $w_{i}$ is the weight of client $i$.

\subsubsection{Network Utility}

Network utility is the sum of the weighted logarithm of the allocated bandwidth of all STAs, which also is the overall channel utilization rate. It is expressed by Equation (4).

$$
U=\sum_{i=1}^{n} w_{i} \log b_{i}
$$

From AP utility, since $b_{i}=\frac{r_{i} \cdot t_{i}}{T_{j}}$, and $r_{i}$ and $T_{j}$ are constants, the network utilization can be expressed by Equation (5).

$$
\begin{gathered}
U=\sum_{i=1}^{n} w_{i} \log t_{i}=\sum_{j=1}^{n}\left(\sum_{i \in A} w_{i} \log t_{i}\right) \\
=\sum_{j=1}^{n} \log \left(\prod_{i \in A}\left(t_{i}\right)^{w_{i}}\right) \\
=\sum_{j=1}^{n} U_{A_{j}}=\log \left(\prod_{j=1}^{n} U_{A_{j}}\right)
\end{gathered}
$$

So, Equation (5) can be transformed into Equation (6).

$$
U \leq n \log \left(\frac{1}{n} \sum_{j=1}^{n} U_{A_{j}}\right)=n \log \overline{U_{A_{j}}}
$$

The equation holds if and only if $U_{A_{1}}=U_{A_{2}}=\cdots=U_{A_{m}}=\overline{U_{A_{j}}}$ when $U=n \log \overline{U_{A_{j}}}$, which means the load of APs is balanced and the channel is utilized fully.

\subsection{Design Requirements}

Based on this network model and performance metrics, load balance guides the design of our algorithm. Simultaneously, we eliminate the performance anomaly problem by exploiting the proportional fairness strategy.

\subsubsection{Load Balance}

Load balance in WLANs is different from that of the cable distributed system, which is mainly embodied to control AP association and handoff, and makes the load balanced among APs [20]. In the IEEE 802.11 standard, the conventional AP association scheme based on RSSI often leads STAs to make associations with congested APs, while leaving adjacent APs to carry very light load or even to be idle, which results in significant bandwidth waste [13]. Thus, some load balance algorithms are often utilized to improve the AP utilization. Therefore, we define an average AP utilization to evaluate the load balance, which is expressed as the following:

$$
\alpha=\frac{\sum_{j=1}^{N}\left(\sum_{i=1}^{M} x_{i j} t_{i j}\right) / T_{j}}{N}
$$

where, $N$ is the number of APs, $M$ is the number of STAs, the coefficient $x_{i j}$ that is a binary variable, indicates the association relationship between STA $i$ and $\mathrm{AP} j, t_{i j}$ denotes that the transmission time of client $i$ obtained in fact from $\mathrm{AP} j$ and $T_{j}$ is the total allocated transmission time of $\mathrm{AP} j$.

\subsubsection{Proportional Fairness}

Proportional fairness is an efficient solution to performance anomaly in multi-rate WLANs. Unlike the legacy DCF mechanism with all clients having the same probability to access the channel, the proportional fairness manner allocates the bandwidth to the clients that are proportional to their 
transmission rates [21]. We assume that there are $m$ clients in the multi-rate WLANs. The set $\left\{b_{1}, b_{2}\right.$, $\left.\ldots, \mathrm{b}_{\mathrm{m}}\right\}$ is the effective bandwidth of client $i$, which is proportionally fair if it is feasible (that is $b_{i} \geq 0$ and $b_{i}=\frac{r_{i} t_{i}}{T_{j}}$ ) and for any other feasible vector $b_{i}^{*}$, the aggregate of proportional changes is either zero or negative, i.e.,

$$
\sum_{i=1}^{m} \frac{b_{i}^{*}-b_{i}}{b_{i}} \leq 0
$$

The network utilization $U\left(b_{i}\right)$ with the bandwidth coefficient is given. It is assumed that a small feasible perturbation $b_{i} \rightarrow b_{i}+\delta b_{i}$ could exist, which increases $U\left(b_{i}\right)$, provided by the following equation.

$$
\sum_{i=1}^{m} \mathrm{U}\left(b_{i}\right)^{\prime} \delta b_{i}>0
$$

According to proportional fairness, Equation (9) can be transformed as follows.

$$
\sum_{i=1}^{m} \frac{\delta b_{i}}{b_{i}}>0
$$

And then the equivalent Equation (11) can be achieved from Equation (10).

$$
\sum_{i=1}^{m}\left(\log b_{i}\right)^{\prime} \delta b_{i}>0
$$

Using the constraints above, the objective function of proportional fairness can be formulated as follows.

$$
\max \sum_{i=1}^{m} \log b_{i}
$$

Ultimately, Equation (12) can be transformed into Equation (13).

$$
\left\{\begin{array}{cl}
\max & \sum_{i=1}^{m} \log t_{i} \\
\text { s.t. } & \sum_{i=1}^{n} t_{i}=T_{j}, \\
& 0 \leq t_{i} \leq T_{j}, \\
& 1 \leq i \leq n .
\end{array}\right.
$$

According to Equation (13), the proportional fairness can be achieved by maximizing the allocated transmission time, which can prevent one client from dominating the channel, thus obtaining the airtime fairness and improving the system throughput.

\section{Bandwidth Allocation and AP Association Algorithms}

In this section, we first present the FBA-BP algorithm to calculate the bandwidth demanded by the clients and try to provide fair service to all clients. Then, we propose the CAA-BD algorithm to solve the performance anomaly problem and achieve the load balance among APs.

\subsection{Bandwidth Allocation Algorithm}

Firstly, we propose the FBA-BP algorithm that allocates bandwidth according to the bandwidth demand of the clients and the clients' business priority. Herein, all clients are divided into four classes according to their application business, and then each class determines the priority in accordance with their demand for bandwidth and delay. As mentioned in Section 3, the priorities are classified into four classes according to their application business. The transmission time $T_{i}$ of client $\mathrm{I}$ is calculated by client demand $S_{i}$, and thus, the total time T for an AP can be achieved by all clients associating with it. Of course, the newly arriving client also needs to be considered. The FBA-BP algorithm can be described in Algorithm 1. 


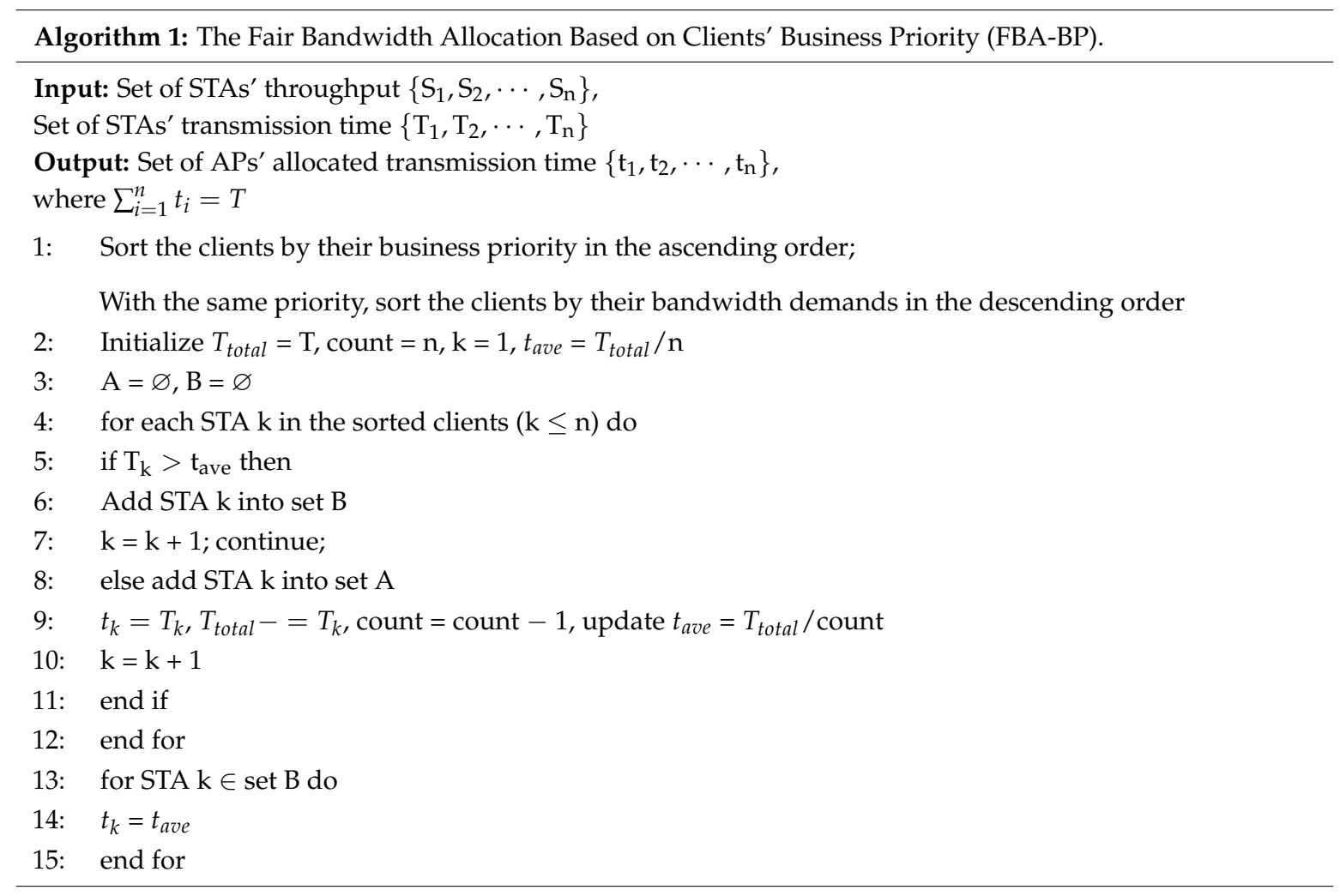

The FBA-BP firstly sorts the clients by the client's business priority. If the clients' business priority is the same, the clients are sorted according to their bandwidth demand. Next, if the demanded time $T_{k}$ of the client $\mathrm{k}$ is equal to or less than the current average allocated time $t_{\text {ave }}$, the client $\mathrm{k}$ also will be added into set $\mathrm{A}$. Otherwise, client $\mathrm{k}$ will be added into set $\mathrm{B}$. Then the current average allocated time is updated with the rest allocable transmission time and the algorithm starts up the next turn until it traverses all the clients associated with the AP. Finally, each client in set B will be allocated with time $t_{\text {ave }}$. Ultimately, Each AP will allocate its transmission time to all its associated clients. Moreover, to meet clients' demands, it also is necessary that the AP should sort the clients by their business priority before allocating its bandwidth.

\subsection{AP Association Algorithm}

In this subsection, we propose a CAA-BD algorithm that first classifies APs by different types of clients associating with them. In addition, then we utilize the aggregated transmission time demanded by the clients as the associating metric of the $\mathrm{AP}$, which can be obtained by FBA-BP algorithm. The CAA-BD algorithm can be described in Algorithm 2. 


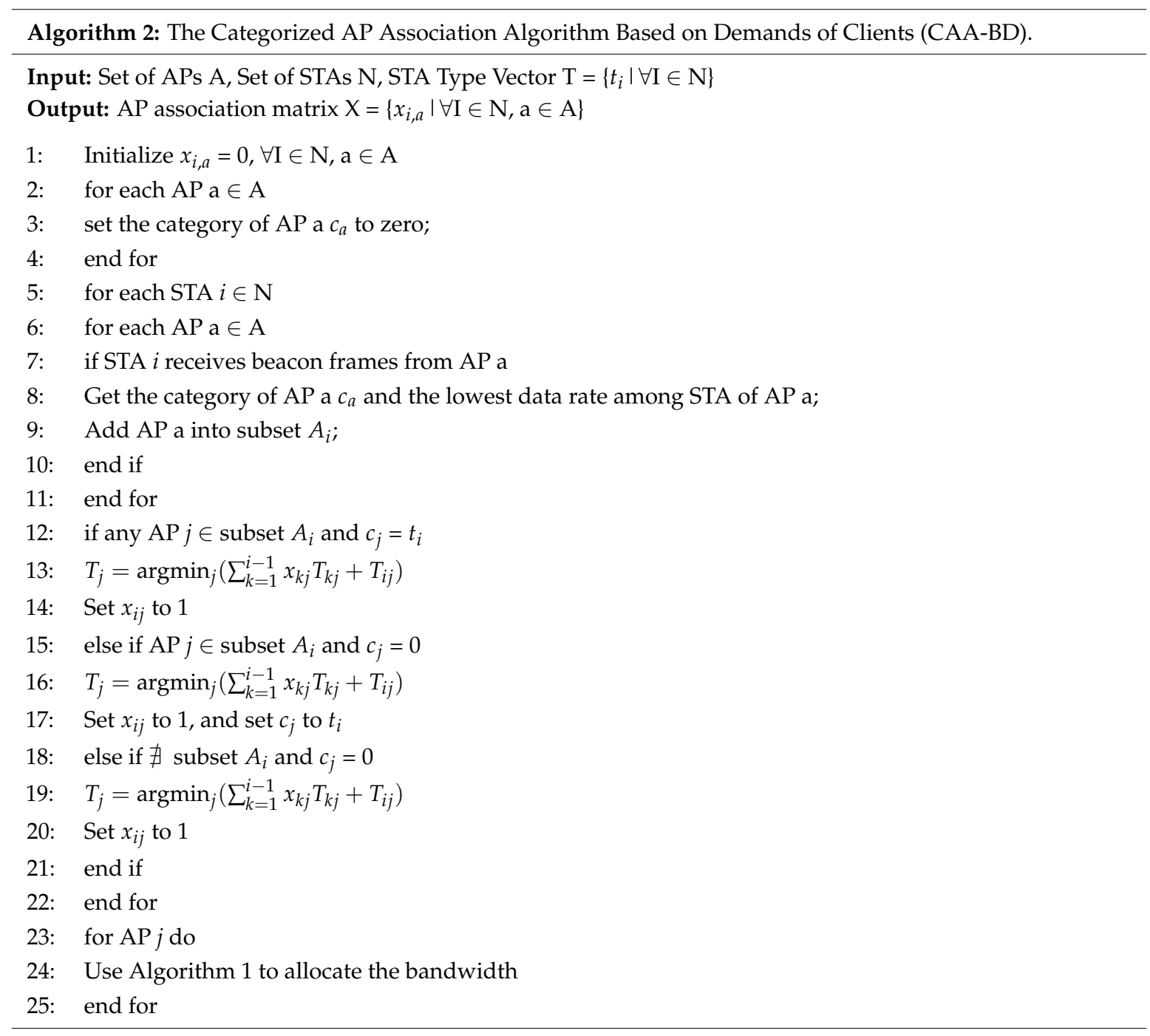

In the pseudo code above, initially, the categories of all APs are set as zero. In addition, then, each AP is classified according to the associated client with the minimum transmission rate, which also may make the transmission rate of all the associated clients close to each other and effectively avoid performance anomaly. Meanwhile, the aggregated transmission time demanded by all clients is used as the metric of AP load when associating, thus the proportional fairness can be achieved among the clients associating to the same AP.

When a new client joins the network and attempts to make an association decision, it firstly acquires a list of candidate APs and potential bit rates according to the signal strength that can be measured by beacons from nearby APs. In addition, then the client selects some APs with the same category based on its bit rate. Finally, the AP with the least allocated transmission time will be chosen as the associating AP. Alternatively, if no such AP exists, the client also may associate with the AP whose category is zero and/or allocated transmission time is the least. Consequently, this strategy can make full use of network resource and effectively improves the utilization of AP and system throughput.

\section{Performance Evaluation}

\subsection{Evaluation Methodology}

In this section, we verify the performance of our algorithm via OPNET 14.5 simulations and compare it with the SSF that is the default AP association scheme in the 802.11 standards and 
Categorized algorithm proposed in [17]. Our simulation considers two typical 802.11 WLANs environments, which are shown in Figure 2. It includes (a) the uniform distribution where all clients are randomly distributed and (b) the hotspot distribution where most of the clients are deployed in a few of the circle-shaped hotspot areas. The network topology of WLAN contains a server, a switch, 20 APs and multiple mobile terminals. The 20 APs are deployed uniformly in $1000 \times 1000 \mathrm{~m}^{2}$ area. While, the density of clients is dynamically changing and its number ranges from 40 to 200 at the interval of 20. Under different network densities, each algorithm simulation runs 30 times, and obtains the average performance for comparison. The coverage area of each AP is set to $150 \mathrm{~m}$ and thus the overlapping area exists between two adjacent APs. Since the transmission rate of the clients is difficult to obtain in realistic deployment, we acquire it by utilizing the relationship between the transmission rate $R_{i j}$ and the Signal to Interference plus Noise Ratio (SINR) $\gamma_{i j}$, which is shown in Table 2 [22].

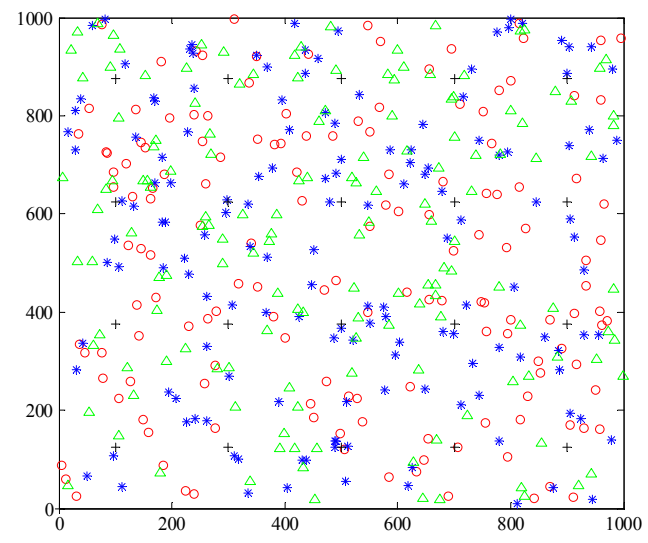

(a)

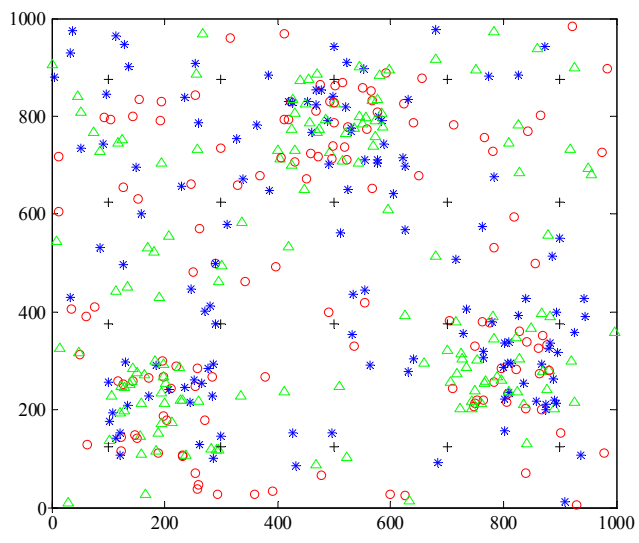

(b)

Figure 2. Network simulation scenarios for different distributions (the different shapes represent $802.11 \mathrm{~b}$ and $802.11 \mathrm{~g}$ clients with different rates and the black plus signs denote APs). (a) The uniform distribution; (b) The hotspot distribution.

Table 2. The relationship between the effective bit rates and the SINRs.

\begin{tabular}{ccccccccc}
\hline$\gamma_{i j}(d B)$ & $6-7.8$ & $7.8-9$ & $9-10.8$ & $10.8-17$ & $17-18.8$ & $18.8-24$ & $24-24.6$ & $>24.6$ \\
\hline$R_{i j}(M b p s)$ & 6 & 9 & 12 & 18 & 24 & 36 & 48 & 54 \\
\hline
\end{tabular}

The performance evaluation metrics include the average AP utilization, system throughput, delay, and the fairness index, where the average AP utilization is calculated by Equation (7). The fairness index that ranges from 0 to 1 and reflects the client fairness in time is defined as follows:

$$
\beta=\frac{\left(\sum_{i=1}^{M} y_{i}\right)^{2}}{M\left(\sum_{i=1}^{M} y_{i}^{2}\right)}, \beta \in[0,1]
$$

where $y_{i}$ is the allocated transmission time for client $i$.

\subsection{Evaluation Results}

\subsubsection{Throughput and AP Utilization}

The aggregated throughputs of three kinds of algorithms are shown in Figures 3 and 4. Both the uniform and hotspot distributions are studied. As is shown, when the number of clients is 40, which means that the load is light and the clients' demands almost are fulfilled, the aggregated throughputs of the three algorithms are very close. With the increasing of client scale, the aggregated 
throughput of SSF algorithm is much lower than that of the other two algorithms, and CAA-BD algorithm can achieve higher throughput than SSF and Categorized algorithm can do, especially, when the number of clients grows beyond 120 in the uniform distribution and beyond 60 in the hotspot distribution. This may be due to the impact of load imbalance among APs for SSF and Categorized algorithm. However, it is alleviated in the CAA-BD algorithm. When the number of clients is 200, the aggregated throughputs of CAA-BD algorithm in the uniform and hotspot distributions are respectively $13.1 \%$ and $32.4 \%$ higher than that of Categorized algorithm. In particular, in Figure 4, when the number of clients is beyond 120, the aggregated throughput of Categorized algorithm decreases drastically, which may be due to the overload of the APs in hotspots distribution. However, The Categorized algorithm does not make a fine-grained association decision.

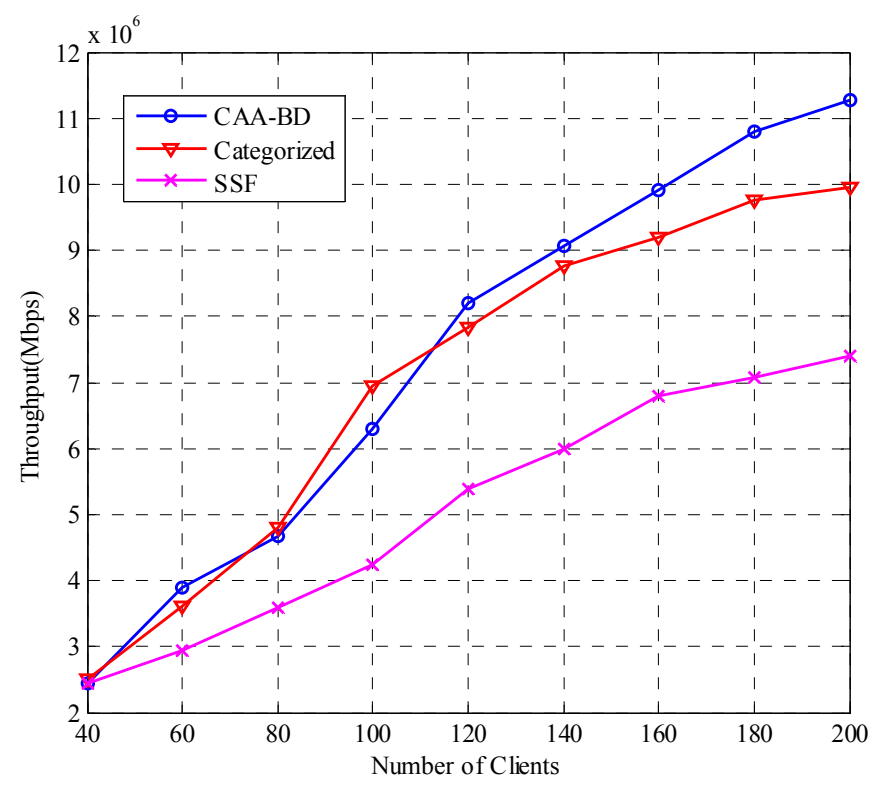

Figure 3. Aggregated throughput in the uniform distribution.

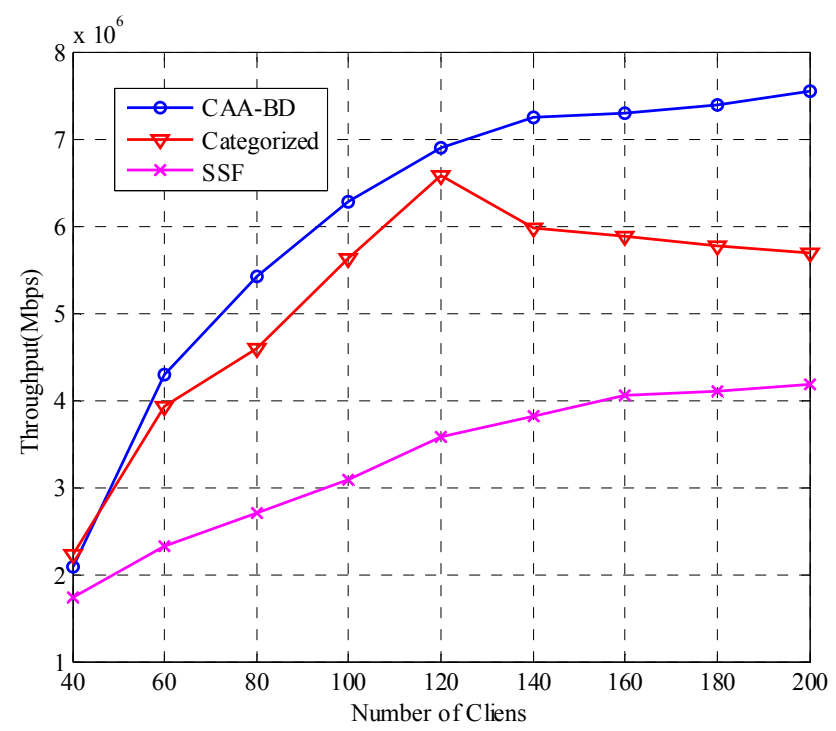

Figure 4. Aggregated throughput in the hotspot distribution. 
The average AP utilizations of all algorithms in the uniform and hotspot distributions are shown in Figures 5 and 6. When the number of clients is 200, the average AP utilization of CAA-BD algorithm gets a significant increase, with $47.9 \%$ in the uniform distribution and $66.6 \%$ in the hotspot distribution respectively, compared with that of Categorized algorithm. It is obvious that the load balance is more significant in CAA-BD algorithm, rather than the other two algorithms. Accordingly, it also illustrates that the average AP utilization for CAA-BD algorithm may result in the high throughput.

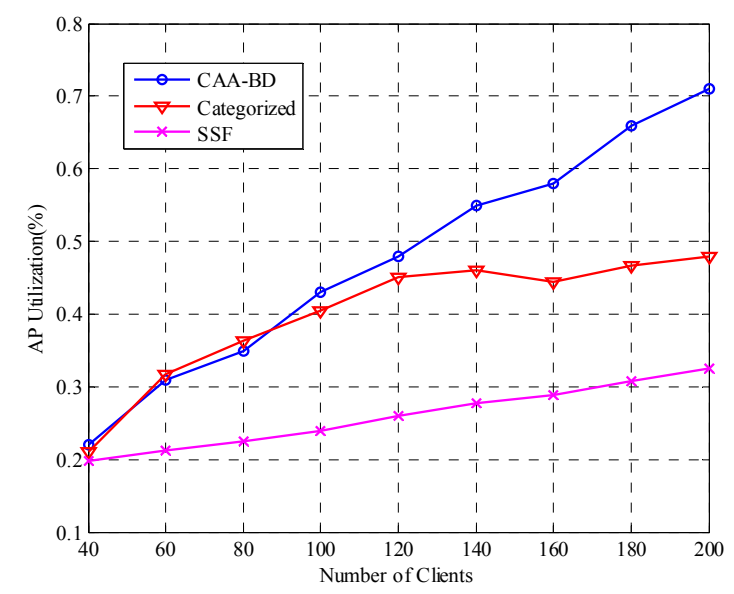

Figure 5. AP utilization in the uniform distribution.

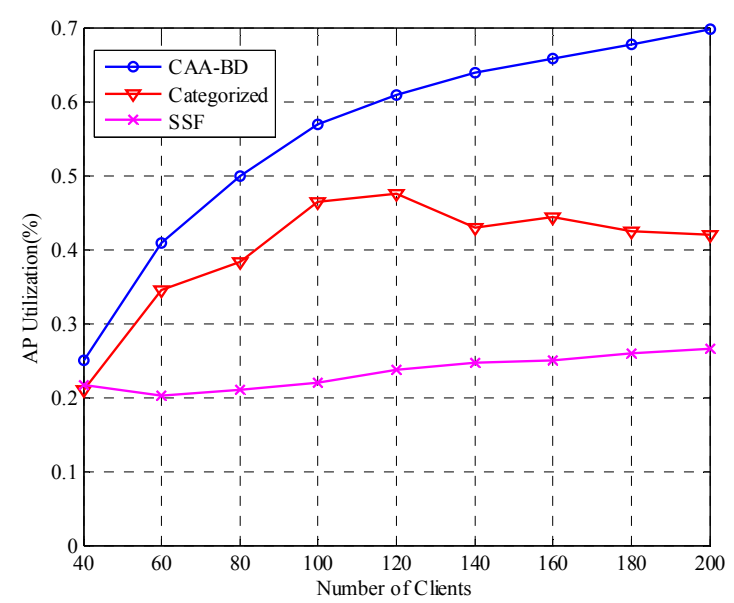

Figure 6. AP utilization in the hotspot distribution.

\subsubsection{Delay}

We also examine the average delay of three kinds of algorithms in the uniform and hotspot distributions. Figures 7 and 8 show the delay increases along with the increase of clients, owing to the higher channel conflict with the client density increasing. When the number of clients is 200, the delay of CAA-BD algorithm gets a $16 \%$ improvement in the uniform distribution and $24.2 \%$ improvement in the hotspot distribution respectively, compared with that of the Categorized algorithm. 


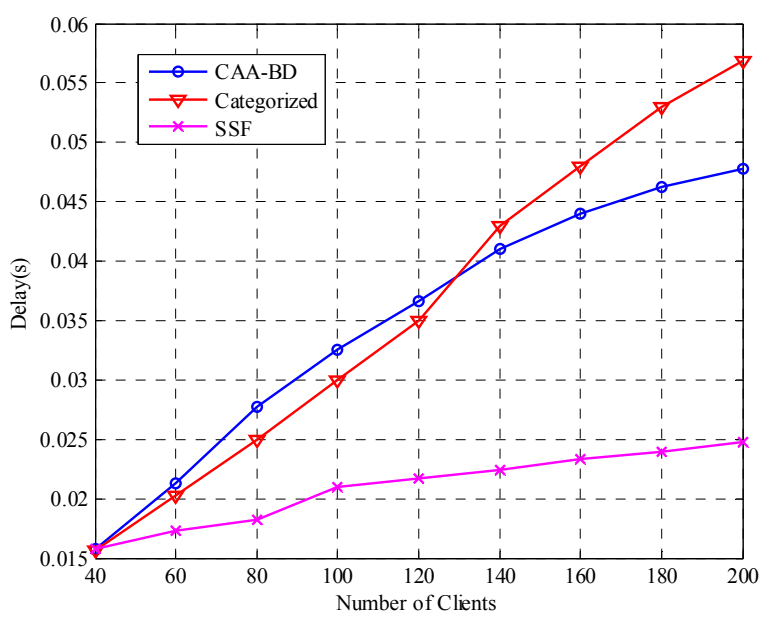

Figure 7. Delay in the uniform distribution.

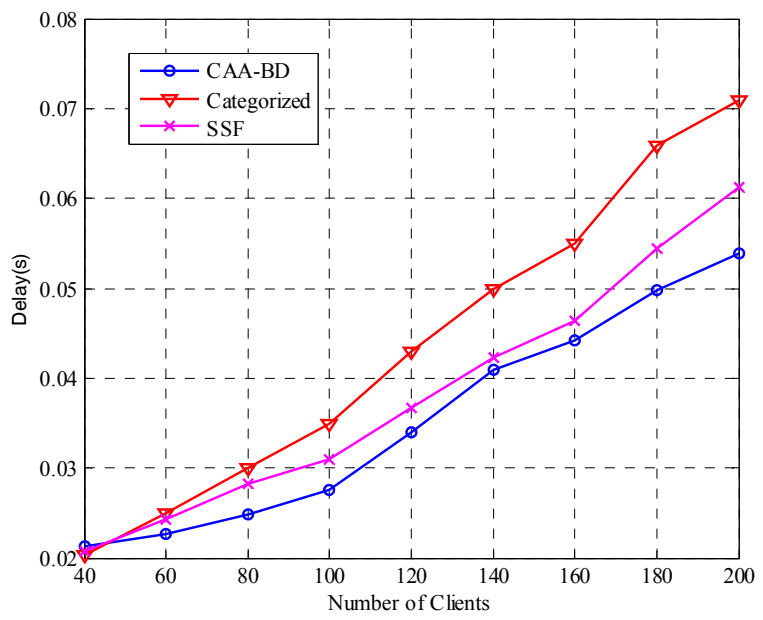

Figure 8. Delay in the hotspot distribution.

Under the uniform distribution, the SSF obtains the shortest delay, which is because many clients can achieve a high transmission rate according to the algorithm associating to the AP with the strongest signal and all APs can almost keep load balance to a certain extent under the uniform distribution scenario. However, the delay of CAA-BD algorithm is the shortest under the hotspot distribution. The reason is that the CAA-BD algorithm can avoid clients gathering, and make full use of APs, which alleviates the conflict partly among clients. While in SSF and Categorized algorithms, some APs are overloaded, and the others are idle or light-loaded, which result in the wasting of some APs. Conversely, the CAA-BD can redistribute some transmission traffic of the overloaded APs to some light-loaded APs.

\subsubsection{Fairness Index}

Finally, we also evaluate the fairness index in terms of transmission time for all algorithms as the client density increases. As shown in Figures 9 and 10, our algorithm obtains higher fairness than the other two algorithms do regardless of the client distribution and density. The fairness index is about $0.8,0.66$ and 0.52 for CAA-BD, Categorized and SSF algorithms under both uniform and hotspot distributions respectively. Obviously, the fairness index for the three algorithms is almost unchanged and has the similar tendency under the uniform distribution, and in the hotspot distribution case, the Categorized algorithm even happens to decline when the number of clients grows beyond 140 . The reason is that the SSF and Categorized algorithms do not show solicitude for load balancing among 
APs. Inevitably, when the client density is high, most of the clients gather on a few APs, which leads to the rest of the APs to be underutilized and wastes network resource.

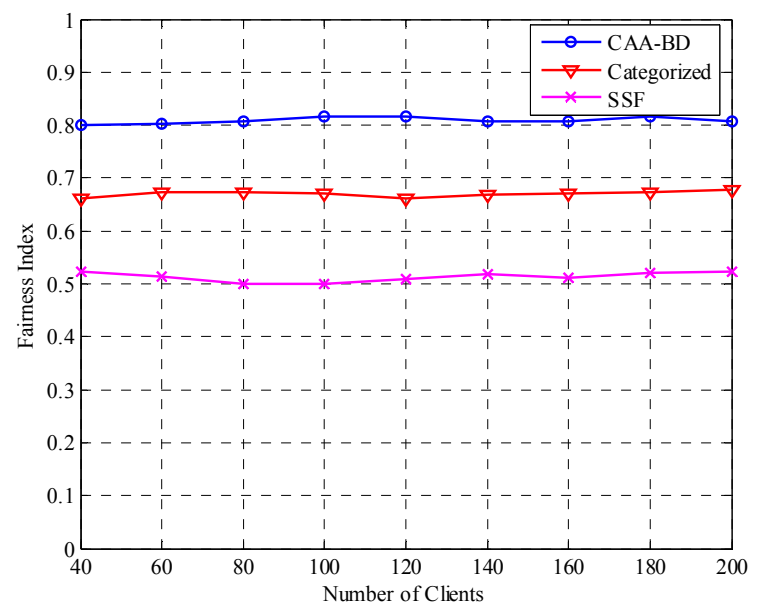

Figure 9. Fairness index in the uniform distribution.

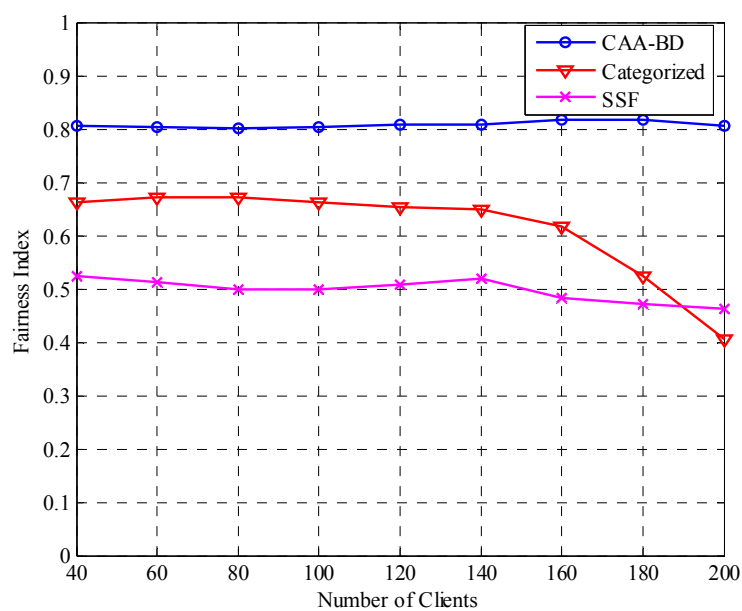

Figure 10. Fairness index in the hotspot distribution.

\section{Conclusions}

In 802.11 WLANs, it is essential that the load balance among APs and fair service to clients are achieved simultaneously. In this paper, we first present the FBA-BP algorithm, which allocates the bandwidth of APs based on their business and priority. Therewith, the aggregated demand time of APs can be calculated according to all the associating clients' bandwidth demands, which is considered as the metric of AP load. Furthermore, we also propose the CAA-BD algorithm that makes a fine-grained association decision based on the categories of APs, as well as AP load whose metric is provided by FBA-BP algorithm. We evaluate the CAA-BD algorithm in terms of average AP utilization, system throughput, delay, and the fairness index under two kinds of deployments. The results show that the CAA-BD can solve the performance anomaly to some extent and obtains load balance simultaneously.

Author Contributions: J.L. contributes to proposing the network model and evaluating methodology; J.T. contributes to presenting the optimized FBA-BP algorithm and performing some evaluating experiments; S.Y. contributes to presenting the CAA-BD algorithm and performing some evaluating experiments.

Funding: This research was supported by the National Science Foundation of China (61602073), National Science Foundation of Chongqing (cstc2014kjrc-qnrc40002) and Fundamental and Advanced Research Project of Chongqing Municipal (cstc2016jcyjA2213). 
Acknowledgments: This paper was presented in part at Springer CSOC2017. The authors would like to thank the anonymous CSOC2017 reviewers for their helpful comments on an earlier version of this work.

Conflicts of Interest: The authors declare no conflict of interest.

\section{References}

1. Liu, Z.; Liu, Y.; Gong, Z.; Chen, L. A Multi-Rate Access Point selection policy in IEEE 802.11 WLANs. In Proceedings of the International Conference on Multimedia Technology (ICMT), Hangzhou, China, 26-28 July 2011; pp. 63-67.

2. Zhang, J.; Bensaou, B. Balancing download throughput in densely deployed IEEE 802.11 multi-cell WLANs. In Proceedings of the 2013 IEEE International Conference on Communications (ICC), Budapest, Hungary, 9-13 June 2013; pp. 6107-6111.

3. Baid, A.; Schapira, M. Network cooperation for client-ap association optimization. In Proceedings of the 2012 10th International Symposium on Modeling and Optimization in Mobile, Ad Hoc and Wireless Networks (WiOpt), Paderborn, Germany, 14-18 May 2012; pp. 431-436.

4. Chen, X.; Cheng, W.; Yuan, W.; Liu, W.; Xu, J. Joint optimization of channel allocation and AP association in variable channel-width WLANs. In Proceedings of the IEEE Wireless Communications and Networking Conference (WCNC), Shanghai, China, 7-10 April 2013; pp. 345-350.

5. Tan, G.; Guttag, J.V. Time-based Fairness Improves Performance in Multi-Rate WLANs. In Proceedings of the 2004 Annual Conference on USENIX Annual Technical Conference, Boston, MA, USA, 27 June-2 July 2004; Volume 12, pp. 187-195.

6. Chen, Z.; Xiong, Q.; Liu, Y.; Huang, C. A strategy for differentiated access service selection based on application in WLANs. In Proceedings of the INFOCOM-IEEE Conference on Computer Communications Workshops, Toronto, ON, Canada, 27 April-2 May 2014; pp. 317-322.

7. Lei, J.; Yang, S.; Su, C. Fairness and Load Balancing Optimization via Association Control in Multi-rate WLANs, Software Engineering Trends and Techniques in Intelligent Systems. In Proceedings of the 6th Computer Science On-Line Conference 2017 (CSOC2017), Princeton, NJ, USA, 26-29 April 2017; pp. $263-275$.

8. Ge, W.; Ji, H.; Leung, V.C.M. Access Point Selection for WLANs with Cognitive Radio: A Restless Bandit Approach. In Proceedings of the IEEE International Conference on Communications, Kyoto, Japan, 5-9 June 2011; pp. 1-5.

9. Pradeepa, B.K.; Kuri, J. An Estimated Delay Based Association Policy for Web Browsing in a Multirate WLAN. IEEE Trans. Netw. Serv. Manag. 2012, 9, 346-358.

10. Keranidis, S.; Korakis, T.; Koutsopoulos, I. Contention and traffic load-aware association in IEEE 802.11 WLANs: Algorithms and implementation. In Proceedings of the International Symposium on Modeling and Optimization in Mobile, Ad Hoc and Wireless Networks, Princeton, NJ, USA, 9-13 May 2011; pp. 334-341.

11. Chen, X.; Yuan, W.; Cheng, W. Access Point Selection under QoS Requirements in Variable Channel-Width WLANs. IEEE Wirel. Commun. Lett. 2013, 2, 114-117. [CrossRef]

12. Wang, S.; Cui, Y.; Xu, K. Multi-Constraint Load Balancing Based on Cell Breathing in WLAN. Chin. J. Comput. 2009, 32, 1947-1956.

13. Gong, H.; Nahm, K.; Kim, J.W. Distributed Fair Access Point Selection for Multi-Rate IEEE 802.11 WLANs. In Proceedings of the 2008 5th IEEE Consumer Communications and Networking Conference, Las Vegas, NV, USA, 10-12 January 2008; pp. 528-532.

14. Bejerano, Y.; Han, S.J.; Li, L. Fairness and Load Balancing in Wireless LANs Using Association Control. IEEE/ACM Trans. Netw. 2007, 15, 560-573. [CrossRef]

15. Li, W.; Cui, Y.; Cheng, X. Achieving Proportional Fairness via AP Power Control in Multi-Rate WLANs. IEEE Trans. Wirel. Commun. 2011, 10, 3784-3792. [CrossRef]

16. Gong, D.; Yang, Y. AP association in 802.11n WLANs with heterogeneous clients. In Proceedings of the 2012 IEEE INFOCOM, Orlando, FL, USA, 25-30 March 2012; pp. 1440-1448.

17. Gong, D.; Yang, Y. On-Line AP Association Algorithms for 802.11n WLANs with Heterogeneous Clients. IEEE Trans. Comput. 2014, 63, 772-786. [CrossRef]

18. Abusubaih, M. On Performance Anomaly in 802.11 Wireless LANs: Problem and Solution Approaches. In Proceedings of the International Conference on Next Generation Mobile Applications. IEEE Computer Society, Amman, Jordan, 27-29 July 2010; pp. 208-212. 
19. Yen, L.; Li, J.; Lin, C. Stability and Fairness of AP Selection Games in IEEE 802.11 Access Networks. IEEE Trans. Veh. Technol. 2011, 60, 1150-1160. [CrossRef]

20. Li, W.; Wang, S.; Cui, Y. AP Association for Proportional Fairness in Multi-rate WLANs. IEEE/ACM Trans. Netw. 2014, 22, 191-202. [CrossRef]

21. Jain, R.; Chiu, D.; Hawe, W. A Quantitative Measure of Fairness and Discrimination for Resource Allocation. In Shared Computer Systems, Computer Science; ACM: New York, NY, USA, 1998.

22. Cui, Y.; Li, W.; Cheng, X. Partially overlapping channel assignment based on "node orthogonality" for 802.11 wireless networks. In Proceedings of the 2011 IEEE INFOCOM, Shanghai, China, 10-15 April 2011; pp. 361-365.

2018 by the authors. Licensee MDPI, Basel, Switzerland. This article is an open access article distributed under the terms and conditions of the Creative Commons Attribution (CC BY) license (http://creativecommons.org/licenses/by/4.0/). 